\title{
Feasibility of cerebello-cortical stimulation for intraoperative neurophysiological monitoring of cerebellar mutism
}

\author{
Davide Giampiccolo ${ }^{1} \cdot$ Federica Basaldella ${ }^{2}$ - Andrea Badari ${ }^{2} \cdot$ Giovanna Maddalena Squintani $^{3} \cdot$ Luigi Cattaneo $^{4}$. \\ Francesco Sala ${ }^{1}$ (D)
}

Received: 12 November 2020 / Accepted: 11 March 2021 / Published online: 9 April 2021

(C) The Author(s) 2021

\begin{abstract}
Background Cerebellar mutism can occur in a third of children undergoing cerebellar resections. Recent evidence proposes it may arise from uni- or bilateral damage of cerebellar efferents to the cortex along the cerebello-dento-thalamo-cortical pathway. At present, no neurophysiological procedure is available to monitor this pathway intraoperatively. Here, we specifically aimed at filling this gap.

Methods We assessed 10 patients undergoing posterior fossa surgery using a conditioning-test stimulus paradigm. Electrical conditioning stimuli (cStim) were delivered to the exposed cerebellar cortex at interstimulus intervals (ISIs) of 8-24 ms prior to transcranial electric stimulation of the motor cortex, which served as test stimulus (tStim). The variation of motor-evoked potentials (MEP) to cStim + tStim compared with tStim alone was taken as a measure of cerebello-cortical connectivity.

Results cStim alone did not produce any MEP. cStim preceding tStim produced a significant inhibition at $8 \mathrm{~ms}(p<0.0001)$ compared with other ISIs when applied to the lobules IV-V-VI in the anterior cerebellum and the lobule VIIB in the posterior cerebellum. Mixed effects of decrease and increase in MEP amplitude were observed in these areas for longer ISIs.

Conclusions The inhibition exerted by cStim at $8 \mathrm{~ms}$ on the motor cortex excitability is likely to be the product of activity along the cerebello-dento-thalamo-cortical pathway. We show that monitoring efferent cerebellar pathways to the motor cortex is feasible in intraoperative settings. This study has promising implications for pediatric posterior fossa surgery with the aim to preserve the cerebello-cortical pathways and thus prevent cerebellar mutism.
\end{abstract}

Keywords Cerebellar mutism $\cdot$ Intraoperative neurophysiological monitoring $\cdot$ Pediatric brain tumours $\cdot$ Posterior fossa

\section{Introduction}

Brain tumours represent the most common solid tumour in the pediatric population, with the majority of them being located in

Luigi Cattaneo and Francesco Sala shared equal contribution as senior author

Francesco Sala

francesco.sala@univr.it

1 Department of Neurosciences, Biomedicine and Movement Sciences, Section of Neurosurgery, University Hospital, Piazzale Stefani 1, 37124 Verona, Italy

2 Intraoperative Neurophysiology Unit, Division of Neurology, University Hospital, Verona, Italy

3 Division of Neurology, University Hospital, Verona, Italy

4 CIMeC-Center for Mind/Brain Sciences, University of Trento, Trento, Italy the posterior fossa [1, 2]. Initially described in 1958 [3], cerebellar mutism $(\mathrm{CM}$, also referred as akinetic mutism or posterior fossa syndrome) includes a variety of signs and symptoms including mutism or speech disturbances, dysphagia, decreased attention and emotional lability. Despite being only anecdotal reported before 1995 [4], recent publication shows that it may occur from 26.6 to $32 \%$ of pediatric patients, with up to $39 \%$ of patients operated for medulloblastoma [5]. While mutism is always transient [6], short-term recovery is incomplete in $98.8 \%$ of patients [7]. Long-term language and cognitive deficits have been documented in a not negligible number of patients [8-10].

The prevalence of non-motor symptoms of CM is consistent with the hypothesis of cerebello-cortical connections responsible for a cerebellar modulation of cognitive functions [11]. While in the past, a role for cerebello-cortical pathways was restricted to motor control, there is now extensive evidence that the cerebellum also exerts non-motor functions [12], with the cerebellar cortex as well as its subcortical nuclei playing a role in cognition 
[12-15]. The leading hypothesis for the occurrence of CM is mono- or bilateral disconnection of the efferent pathway from the cerebellum to the cortex, namely the cerebello-dentothalamo-cortical (CDTC) pathway [16]. This has been supported by a vast number of recent neuroimaging as well as lesion studies, showing that CM is associated with large [17], midline [18] tumours residing in proximity of the fourth ventricle and predicted by damage of the superior cerebellar peduncles, the dentate nucleus or the CDTC pathway itself [19]. Although tractography can improve preoperative surgical planning or predict postoperative deficit [19], its intraoperative reliability is limited by tissue displacement and brain-shift phenomena [20]. Currently, no intraoperative method exists that can provide a mean to CDTC pathway preservation.

We recently reviewed all intraoperative studies aimed at mapping the cerebellum, and, while CM has been extensively explored using neuroimaging techniques, neurophysiological reports are lacking [21]. This may be surprising, considering that intraoperative neurophysiological monitoring (IONM) is particularly well developed for other posterior fossa surgeries. However, as the cerebello-dento-thalamo-cortical pathway is polysynaptic, it differs from direct motor connections which are less influenced by anaesthesia [22]. One way to assess the CDTC circuit has been provided by non-invasive brain stimulation. Cerebellar stimulation by means of transcranial electrical stimulation (TES) or by transcranial magnetic stimulation (TMS) modulates the amplitude of motor-evoked potentials (MEPs) following TMS of the motor cortex [23]. Such modulation is commonly inhibitory-though it can take the form of excitation with appropriate TMS parameters - and is commonly referred to as cerebellar inhibition (CI) [23]. The latency at which such inhibition occurs is of 5-8 ms, with cerebellar stimulation as conditioning stimulus (cStim) occurring before the test stimulus (tStim) over the motor cortex. CI is commonly thought to be a result of activity along the CDTC pathway [23, 24]. Noticeably, this phenomenon is absent in cases of damage to the CDTC pathway, such as a lesion to the cerebellar hemispheres, the dentate nucleus, the superior cerebellar peduncle and the motor thalamus [25]. We speculate that the adoption of a similar paradigm intraoperatively may offer a method to identify, and possibly prevent, cerebellodento-thalamo-cortical pathway disconnection and therefore cerebellar mutism [21]. With this aim, we explored the feasibility of using an intraoperative paired cerebello-cortical stimulation in ten patients undergoing posterior fossa surgery.

\section{Methods}

\section{Patients' cohort}

The study proposal is in accordance with ethical standards of the Declaration of Helsinki. All stimulations and recordings were performed in the context of clinical intraoperative neurophysiological monitoring (IONM). Patients scheduled for posterior fossa surgery were screened for enrolment and signed a written consent to adhere. The inclusion criteria were (1) posterior fossa disease with indication to intraoperative neurophysiological monitoring. Exclusion criteria were (1) voluntary decision of the patient or his/her family not to be included in the cohort. Ten patients (age 6-73; 5M-5F; 10 right-handed, 2 children) were included in this study. Patient's characteristics are presented in Table 1.

\section{Stereotaxic neuronavigation and electrode placement}

MRI scans of each patient's brain were acquired before surgery on a $1.5 \mathrm{~T}$ or $3 \mathrm{~T}$ scanner with an eight-channel head coil (Signa 3T, General Electric Healthcare, Milwaukee, USA). T1-weighted 3D MPRAGE images were acquired using the following parameters (echo train length: 1, TE: $2.67 \mathrm{~ms}$, TR: 2.000, matrix size: $256 \times 246$, slice thickness: $1 \mathrm{~mm})$. T2weighted, FLAIR images were also acquired (TR $6000 \mathrm{~ms}$, TE $150 \mathrm{mss}$, TI $2000 \mathrm{~ms}$ ). The reconstruction of the individual cortical surface was performed using Brainsuite (Brainsuite [26], UCLA Brain Mapping Center, San Francisco, USA). For a clearer intraoperative visualization of sulcal anatomy, a skull stripped T1 using a non-uniformity correction or FLAIR images was added to the $3 \mathrm{D}$ visualization of the Neuronavigation system (Stealth Station 7, Medtronic, Minneapolis, USA). Correspondence of 3D reconstruction and individual patient's sulcal anatomy was then performed using the Neuronavigation pointer. Brain anatomy was systematically analysed prior to surgery so that the main sulcal pattern of the principal sulcus as well as the vermis could be identified during surgery. Placement of the conditioning electrode strip was planned a priori but was systematically reprogrammed when in presence of contingent surgical conditions preventing the placement of the strip in the desired position, such as presence of large vessels or space requirements by the ongoing surgical procedures.

\section{Anaesthesia and conventional IONM}

The anaesthesia protocol applied was Total IntraVenous Anaesthesia (TIVA). More precisely, a continuous infusion of Propofol (100-150 $\mu \mathrm{g} / \mathrm{kg} / \mathrm{min})$ and Fentanyl $(1 \mu \mathrm{g} / \mathrm{kg} /$ min) was used, avoiding bolus. Halogenated anaesthetic agents were never used. Since all patients were candidates for IONM of the corticospinal tract, standard neurophysiological monitoring and mapping were performed. This involved simultaneous acquisition of continuous electroencephalography (EEG) and recording of free-running electromyographic (EMG) activity (ISIS-IOM, Inomed Medizintechnik GmbH, Emmendingen, Germany). Muscle MEPs were elicited by transcranial electrical stimulation (TES) via corkscrew-like 
Table 1 Patient demographics

\begin{tabular}{llllll}
\hline Patient & Age & Sex & Symptoms & Diagnosis & Surgical approach \\
\hline$\# 1$ & 73 & F & Incidental & Tentorial meningioma (WHO I) & RS \\
$\# 2$ & 47 & F & Vertigo & Tentorial meningioma (WHO I) & MS \\
$\# 3$ & 8 & M & Hydrocephalus & Medulloblastoma (WHO IV) & MS \\
$\# 4$ & 23 & M & Cephalalgia & Chiari malformation I & MS \\
$\# 5$ & 37 & M & Diplopia & Glio-neuronal neoplasia of the lamina quadrigemina (WHO III) & MS \\
$\# 6$ & 37 & M & Vertigo & Hemangioblastoma in VHL & RS \\
$\# 7$ & 50 & M & Vertigo & Hemangioblastoma in VHL & RS \\
$\# 8$ & 61 & F & Hear loss & Vestibular schwannoma (WHO I) & MS \\
$\# 9$ & 18 & F & Cephalalgia & Hemangioblastoma in VHL & MS \\
\hline 10 & 9 & M & Hydrocephalus, diplopia & Pilocytic astrocytoma (WHO I) & \\
\hline
\end{tabular}

$M S$ median suboccipital approach, $R S$ retrosigmoid approach, $V H L$ Von Hippel-Lindau syndrome

electrodes (Ambu Neuroline Corkscrew, Ambu, Copenhagen, Denmark) from the scalp. Short trains of 5 square-wave stimuli of $0.5 \mathrm{~ms}$ duration and interstimulus interval (ISI) of $2 \mathrm{~ms}$ were applied at a repetition rate up to $2 \mathrm{~Hz}$ through electrodes placed at C1 and C2 scalp sites, according to the 10/20 EEG system [27].

\section{Motor stimulation as test stimulus}

TES was applied to precentral gyrus (test stimuli) via a C1-C2 dipole on the skull using corkscrew (Ambu Neuroline Corkscrew, Ambu, Copenhagen, Denmark) (Fig. 1 shows a schematic of the dual stimulation protocol and an example of surgical scenario). Stimulation parameters were personalised in each patient and set to obtain a MEP from the thenar muscle of around $500 \mu \mathrm{V}$ peak-peak amplitude (see Table 2). As a result, stimuli were delivered with trains of 2-5 stimuli in different patients, at an intrinsic frequency of $500 \mathrm{~Hz}$ at an intensity of 75-200 mA (Table 2).

\section{Cerebellar stimulation}

The cerebellar cortex was stimulated with a short train of stimuli at $500 \mathrm{~Hz}$ ranging from 2 (To2) to 5 (To5), with current intensity of 15-25 mA (Table 2). Stimuli were delivered through an electrode strip (contact diameter: $5 \mathrm{~mm}$, interelectrode distance of $10 \mathrm{~mm}$, contact strips: $0.7 \mathrm{~mm}$ thin, $10 \mathrm{~mm}$ width, EB Neuro S.p.A., Firenze, Italy) placed directly on the cerebellar cortex where the cortex was exposed and gently slipped under the dura outside the craniotomic window. Bipolar stimulation was delivered using pairs of adjacent electrodes. The number of effective contacts varied between patients according to surgical needs.

Table 2 Stimulation parameters

\begin{tabular}{llllllll}
\hline Patient & $\begin{array}{l}\text { No } \\
\text { pulses }\end{array}$ & $\begin{array}{l}\text { TES } \\
(\mathrm{mA})\end{array}$ & $\begin{array}{l}\text { DCS } \\
(\mathrm{mA})\end{array}$ & $\begin{array}{l}\text { MEP from } \\
\text { direct } \\
\text { cerebellar } \\
\text { DCS }\end{array}$ & $\begin{array}{l}\text { Pre- } \\
\text { operative } \\
\text { MRC }\end{array}$ & $\begin{array}{l}\text { Post- } \\
\text { operative } \\
\text { MRC }\end{array}$ & $\begin{array}{l}\text { Follow-up } \\
\text { MRC }\end{array}$ \\
\hline$\# 1$ & To3 & 140 & 20 & no & 5 & 5 & 5 \\
$\# 2$ & To4 & 180 & 15 & no & 5 & 5 & 5 \\
$\# 3$ & To5 & 180 & 25 & no & 5 & 2 & 5 \\
$\# 4$ & To5 & 160 & 20 & no & 5 & 5 & 5 \\
$\# 5$ & To5 & 200 & 20 & no & 5 & 5 & 5 \\
$\# 6$ & To5 & 200 & 20 & no & 5 & 5 & 5 \\
$\# 8$ & To2 & 180 & 20 & no & 5 & 5 & 5 \\
$\# 9$ & To3 & 90 & 20 & no & 5 & 5 & 5 \\
$\# 10$ & To4 & 75 & 20 & no & 5 & 5 & 5 \\
\hline
\end{tabular}

TES transcranial electrical stimulation, DCCS direct cerebellar cortical stimulation, $M R C$ Medical Research Council 

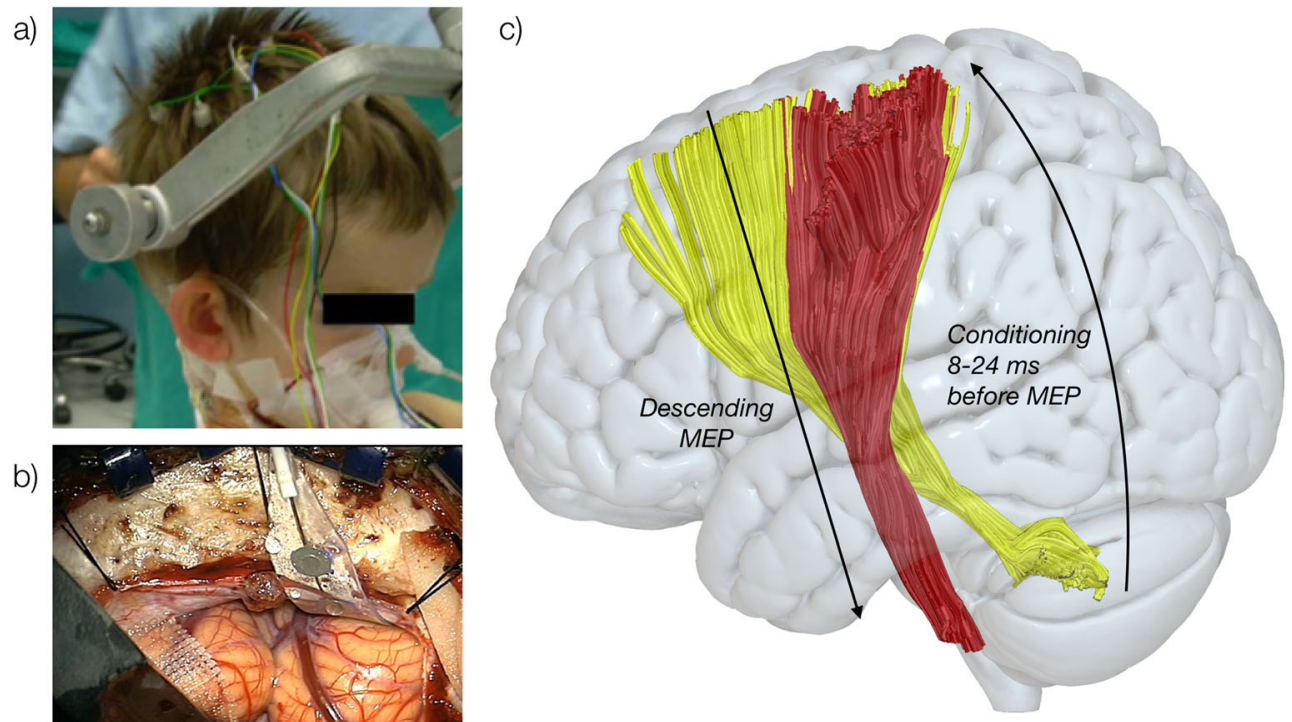

b)

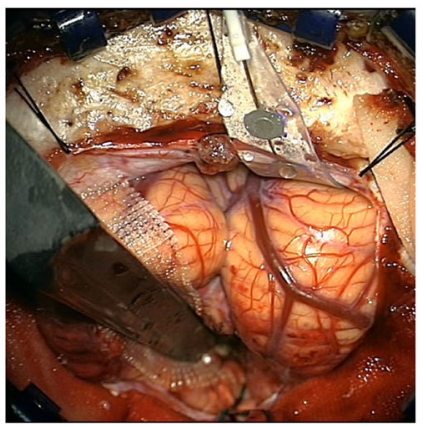

Fig. 1 Schematic representation of the stimulation protocol. a) M1 stimulation (test stimulus) is performed using transcranial electric stimulation using a C1-C2 dipole on the skull, which generates motorevoked potentials by activating the corticospinal tract. b) Direct cerebellar stimulation is applied as conditioning stimulus prior to M1 stimulation, causing MEP modulation. c) Representation of stimulation protocol:

\section{Paired stimulation protocol}

Paired stimulation was compared with M1-only stimulation. Briefly, we acquired 20 stimulation from transcranial electric stimulation of M1, which were considered as baseline stimuli. Then, other 20 stimuli were acquired directly stimulating the cerebellum alone, in order to exclude any direct MEP result arising from the cerebellum. To conclude, we performed 20 paired stimulations where the cerebellum was stimulated before M1. M1 stimulation occurred therefore after cerebellar stimulation at fixed interval between 8 and $24 \mathrm{~ms}$. The timing of dual stimuli was managed entirely by the commercially available ISIS-IOM system (Inomed Medizintechnik GmbH, Emmendingen, Germany) by means of the "facilitation" function, which allows independent electrical stimulation through two separate output channels.

\section{Data analysis}

Data analysis is explained in detail in the Supplementary Material. Briefly, paired stimulation was compared with M1 only stimulation (baseline stimulation) in the single patient. Preprocessing required the data to be exported in digital format and analysed with MATLAB software. The EMG traces were band-pass filtered (10-3000 Hz) and positive peak-to-peak amplitude was extracted from each MEP. As data were not normally

direct cerebellar precedes transcranial electrical stimulation with an inter-stimulus interval (ISI) between 8 and $24 \mathrm{~ms}$. Conditioning stimuli generating MEP facilitation/inhibition compared with test stimuli alone are considered evidence for functional connectivity between the two stimulated regions. Red: corticospinal tract; yellow: cerebello-dentothalamo-cortical pathway

distributed in blocks of stimuli, a non-parametric MannWhitney's $U$ test was performed. Paired stimulation that leads to a significant increase in MEP compared with baseline was considered excitatory, while those that lead to a significant decrease in MEP were considered inhibitory. The significance threshold for the $p$ value was set to 0.005 rather than performing actual multiple comparison correction, as the number of conditions (strip electrodes and ISIs) was not the same in all patients, and accordingly, the number of multiple comparisons varied among participants. Individual cerebellar anatomy and stimulation sites acquired during neuronavigation were normalised to the MNI space using SUIT[28] (http://www.diedrichsenlab. org). Since not all stimulation sites could be acquired with neuronavigation because it is not exposed in the craniotomy, the location of each subdural electrode was reconstructed using 3D Slicer (www.slicer.org) using the strip electrode trajectory on individual cerebellar surface considering a fixed interelectrode distance of $1 \mathrm{~cm}$. MNI coordinates of stimulation sites per individual patient can be found in Table 3 .

\section{Results}

In all participants, it was possible to stimulate at least one conditioning dipole. Figure 2 shows each patient's anatomy together with lesion location and electrode placement while 
Table 3 MNI location of all recording contacts

\begin{tabular}{|c|c|c|c|c|}
\hline \multirow{2}{*}{$\begin{array}{l}\text { Patient } \\
\# 1\end{array}$} & \multirow{2}{*}{$\begin{array}{l}\begin{array}{l}\text { Recording } \\
\text { electrodes }\end{array} \\
\text { EL }\end{array}$} & \multicolumn{3}{|c|}{ MNI coordinates } \\
\hline & & $x$ & $y$ & $z$ \\
\hline & 1 & 51 & -50 & -51 \\
\hline & 2 & 51 & -61 & -51 \\
\hline & 3 & 47 & -71 & -50 \\
\hline & 4 & 40 & -80 & -46 \\
\hline \multirow[t]{5}{*}{$\# 2$} & EL & $x$ & $y$ & $z$ \\
\hline & 1 & 25 & -88 & -24 \\
\hline & 2 & 24 & -91 & -31 \\
\hline & 3 & 19 & -92 & -39 \\
\hline & 4 & 14 & -89 & -45 \\
\hline \multirow[t]{5}{*}{$\# 3$} & EL & $x$ & $y$ & $z$ \\
\hline & 1 & 26 & -57 & -15 \\
\hline & 2 & 26 & -67 & -16 \\
\hline & 3 & 24 & -77 & -18 \\
\hline & 4 & 23 & -85 & -21 \\
\hline \multirow[t]{5}{*}{ \#4 } & EL & $x$ & $y$ & $z$ \\
\hline & 1 & 18 & -65 & -11 \\
\hline & 2 & 21 & -72 & -15 \\
\hline & 3 & 25 & -80 & -19 \\
\hline & 4 & 30 & -88 & -24 \\
\hline \multirow[t]{7}{*}{$\# 5 \mathrm{a}$} & EL & $x$ & $y$ & $z$ \\
\hline & 1 & 5 & -76 & -49 \\
\hline & 2 & 12 & -73 & -57 \\
\hline & 3 & 20 & -69 & -61 \\
\hline & 4 & 30 & -63 & -64 \\
\hline & 5 & 36 & -54 & -63 \\
\hline & 6 & 38 & -44 & -57 \\
\hline \multirow[t]{5}{*}{$\# 5 \mathrm{~b}$} & EL & $x$ & $y$ & $z$ \\
\hline & 1 & 17 & -92 & -31 \\
\hline & 2 & 27 & -91 & -32 \\
\hline & 3 & 37 & -87 & -32 \\
\hline & 4 & 45 & -81 & -33 \\
\hline \multirow[t]{4}{*}{$\# 6 a$} & EL & $x$ & $y$ & $z$ \\
\hline & 1 & 26 & -47 & -15 \\
\hline & 2 & 32 & -57 & -18 \\
\hline & 3 & 39 & -66 & -20 \\
\hline \multirow[t]{3}{*}{$\# 6 \mathrm{~b}$} & EL & $\mathrm{x}$ & $\mathrm{y}$ & z \\
\hline & 1 & 16 & -89 & -45 \\
\hline & 2 & 12 & -91 & -37 \\
\hline \multirow[t]{4}{*}{$\# 7$} & EL & $x$ & $y$ & $z$ \\
\hline & 1 & 16 & -49 & -9 \\
\hline & 2 & 20 & -59 & -10 \\
\hline & 3 & 23 & -67 & -15 \\
\hline \multirow[t]{5}{*}{$\# 8$} & EL & $x$ & $y$ & $z$ \\
\hline & 1 & 18 & -42 & -11 \\
\hline & 2 & 21 & -52 & -12 \\
\hline & 3 & 24 & -62 & -14 \\
\hline & 4 & 28 & -71 & -18 \\
\hline
\end{tabular}

Table 3 (continued)

\begin{tabular}{lllll}
\hline Patient & $\begin{array}{l}\text { Recording } \\
\text { electrodes }\end{array}$ & \multicolumn{3}{l}{ MNI coordinates } \\
\hline \multirow{4}{*}{ \#9 } & 5 & 33 & -80 & -21 \\
& EL & $x$ & $y$ & $z$ \\
& 1 & 9 & -78 & -54 \\
& 2 & 17 & -77 & -58 \\
& 3 & 25 & -72 & -61 \\
& 4 & 32 & -65 & -63 \\
& 5 & 37 & -56 & -62 \\
& EL & $x$ & $y$ & $z$ \\
& 1 & 18 & -42 & -11 \\
& 2 & 21 & -52 & -12 \\
& 3 & 24 & -62 & -14 \\
& 4 & 28 & -71 & -18 \\
& 5 & 33 & -80 & -21 \\
& 5 & 18 & -42 & -11 \\
\hline
\end{tabular}

NB. Each patient's second strip positioning in a is signalled as a "b"

Fig. 3 shows each electrode site normalised to the MNI space and visualised on the right side. Intensity of stimulation and conditioning as well as clinical outcome is shown in Table 2.

\section{Direct cerebellar stimulation alone}

No MEP was evoked with direct cerebellar stimulation in any of the patients.

\section{Paired cortico-transcortical stimulation}

We observed significant modulation of MEPs in 8/10 patients. Five patients showed MEP inhibition, 1 patient MEP facilitation and 2 patients showed both conditions at different ISIs. The individual results are reported in the Supplementary Table 1. Coordinates for strip electrodes normalised to MNI can be found in Table 3 .

\section{Anatomical localization of conditioning effects}

Group analysis was not quantitatively performed, and therefore, any observation on the anatomical location of effective cerebellar stimulations is descriptive. Overall, two clusters of response were evidenced: a larger cluster occupying the intermediate portion of lobule IV, V and VI in the anterior cerebellum, and a smaller cluster in the posterior cerebellum occupying the intermediate pre-pyramidal fissure and lobule VIIB. When comparing inhibitory and facilitatory effects in the anterior cerebellum, these seemed to overlap in the anterior cerebellum, though inhibitory responses seemed to be more 
Fig. 2 Rendering of the individual cerebelli. The projection of the lesion on the surface is displayed in yellow. The individual stimulation sites are shown in green (note that conditioning stimuli have been delivered in bipolar modality)
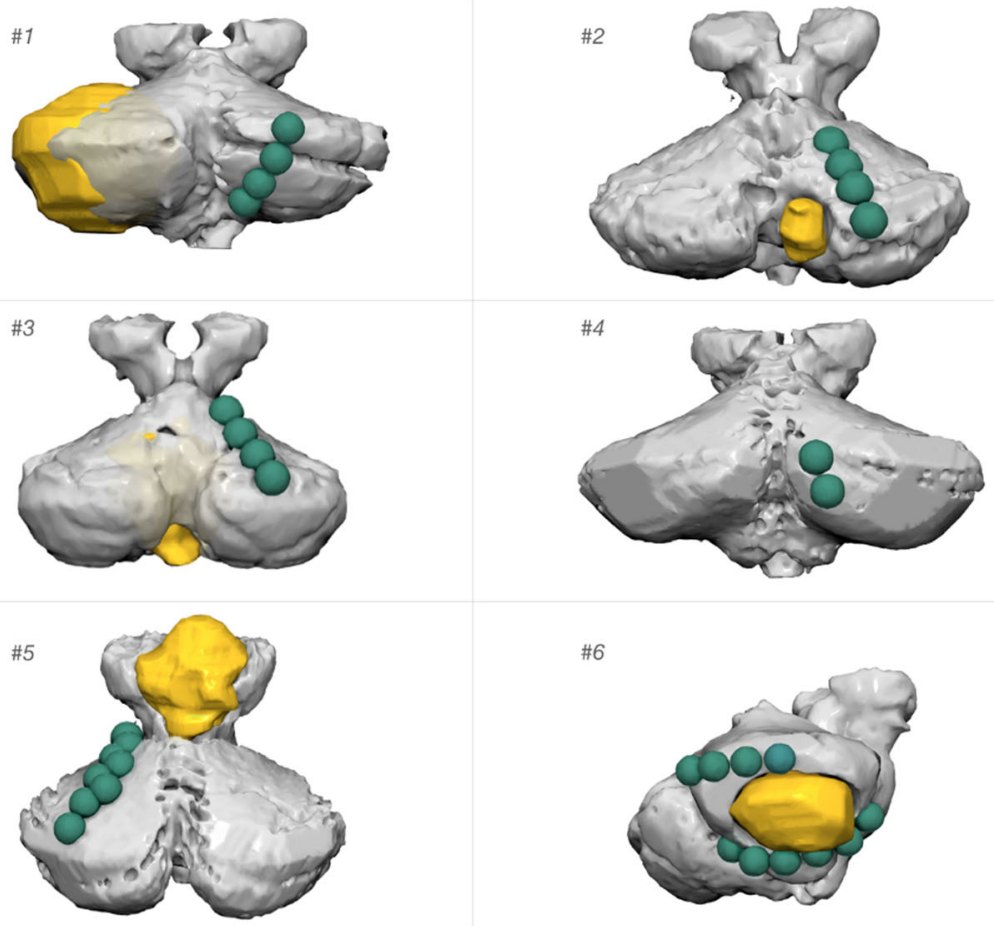

\#6
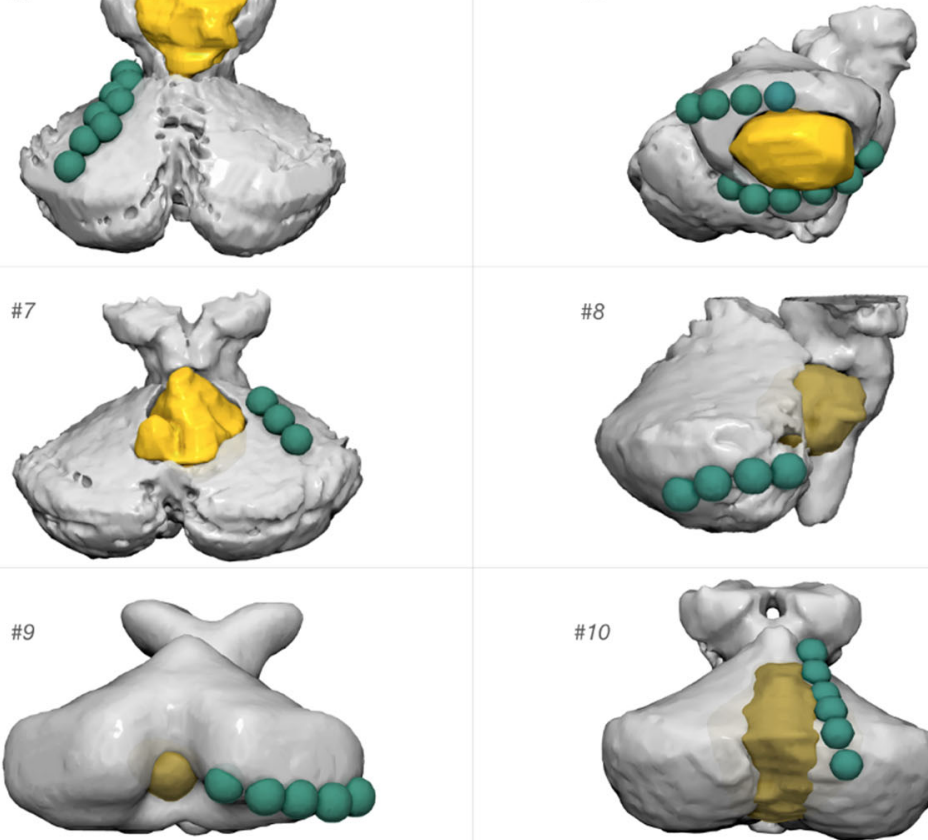

\#8
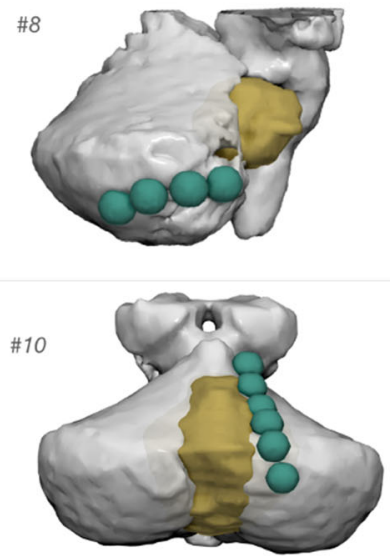

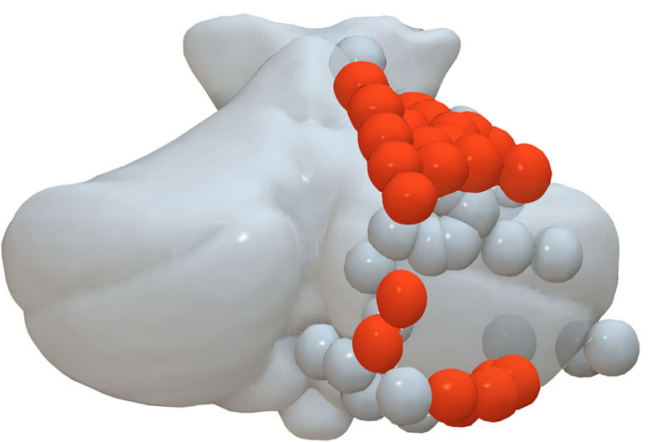

Fig. 3 Normalised data from all patients. All sites are displayed on the right hemisphere. Effective sites are shown in orange, occupying the intermediate portion of lobule IV, V, VI and VIIB. Ineffective sites are shown in grey widespread in both clusters. In the posterior cerebellum, no sites for MEP facilitation were evidenced.

\section{Chronometry of conditioning effects}

Conditions of significant inhibition or excitation were not univocally associated with a single ISI, though a prevalence for the $8 \mathrm{~ms}$ ISI was observed. Inhibitory responses were found overall in 14/152 positions (9\%). These were distributed between different ISIs as follows: in 3/13 (23\%) positions at the $8 \mathrm{~ms}$ ISIs, in $2 / 36(6 \%)$ positions at the $12 \mathrm{~ms}$ ISI and at $5 / 36$ positions (14\%) at the $16 \mathrm{~ms}$ ISI, in $3 / 33$ positions (9\%) at the $20 \mathrm{~ms}$ ISI and in $1 / 26$ positions $(4 \%$ ) at the $24 \mathrm{~ms}$ ISI. Excitatory responses were much less frequent, appearing only in $4 / 152$ positions, none of which at $8 \mathrm{~ms}$ and one for each of the other 4 ISIs. The group analysis yielded significant results 
$(\mathrm{H}(4, N=144)=11.57 p=0.021)$. The results are indicated in Fig. 4. Post hoc exploration of the significant results was carried out with Mann-Whitney's $U$ tests, comparing the data from each ISI reciprocally. The results indicated that the main effect was entirely due to the $8 \mathrm{~ms}$ ISI having lower values than all other ISIs. In particular, the $8 \mathrm{~ms}$ ISI was significantly different from the $12 \mathrm{~ms}$ ISI $(Z=-2.93 ; p=0.003)$, from the $16 \mathrm{~ms}$ ISI $(Z=-3.04$; $p=0.002)$, from the $20 \mathrm{~ms}$ ISI $(Z=-2.66 ; p=0.008)$ and from the $24 \mathrm{~ms}$ ISI $(Z=-2.81 ; p=0.004)$. All other reciprocal comparisons between all other ISIs were non-significant. Finally, as a second post hoc comparison, we sought to understand whether the data from each ISI was significantly different from a distribution centred on a mean value of $x=0$. The significance of this test was to assess whether stimulation at each ISI yielded significant inhibition, no effect or facilitation. The results showed that the $z-$ values in the $8 \mathrm{~ms}$ ISI were highly significantly smaller than $x=0$ $(t(12)=-5.57 ; p=0.0001)$, corresponding to MEP inhibition, while at all other ISIs, no effect was observed $(\min p$ value $=0.17)$.

The anatomical location of the conditioning spots that exerted a modulation on the MEPs is illustrated in Fig. 5. Qualitative inspection seemed to indicate defined clusters for the different ISIs. In particular, a medio-lateral distribution of conditioning responses was shown in the anterior cerebellum: $8 \mathrm{~ms}$ responses occurred medially, $12 \mathrm{~ms}$ were central and $16 \mathrm{~ms}$ responses lateral. Similarly, $20 \mathrm{~ms}$ lied medially to those at $24 \mathrm{~ms}$. Responses in the posterior cerebellum showed mixed distribution (Fig. 6).

\section{Discussion}

We explored the feasibility of intraoperative dual cerebellomotor stimulation with the aim of developing an

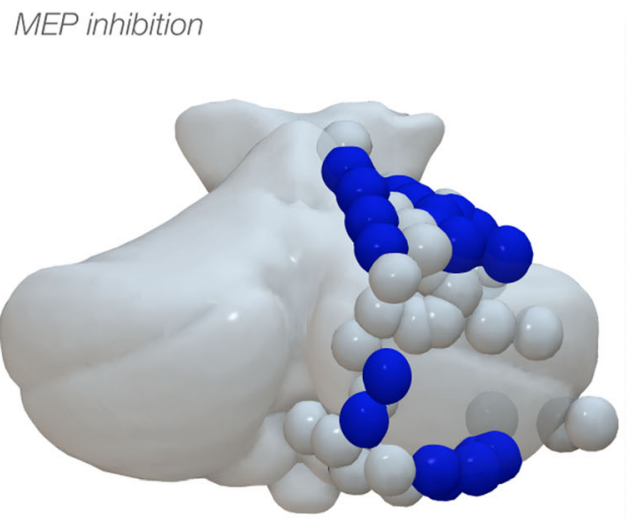

Fig. 4 Standardised cerebellar anatomy showing the location of each participant's conditioning electrodes in the cerebellar cortex. All stimulation sites have been displayed on the right hemisphere as spheres. The left panel shows the cerebellar location of dipoles with inhibitory effect over M1, occurring when conditioning over the intermediate lobule IV-V-VI in the anterior cerebellum or the lobule

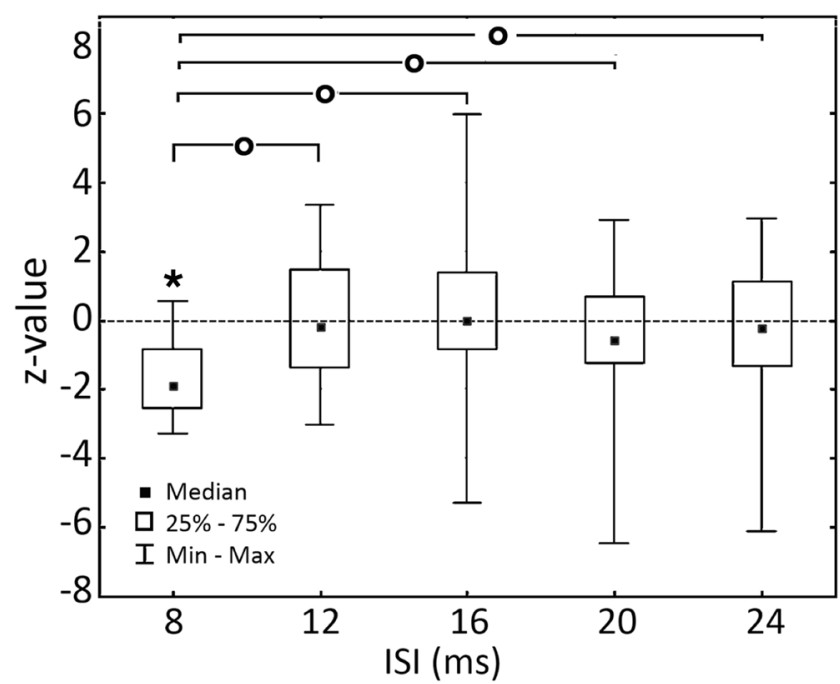

Fig. $5 z$ values from the whole population of patients, grouped by ISI. Circles indicate significant differences between ISIs. The asterisk indicates the significant difference between the data in each ISI and the null hypothesis of a distribution centred on $x=0$

intraoperative method to monitor, and therefore preserve, the cerebello-dento-thalamo-cortical pathway, a principal network involved in cerebellar mutism. To do this, we reproduced the extraoperative conditioning-test paradigm developed by Ugawa and colleagues [23] intraoperatively.

In agreement with previous extraoperative literature [23], our data showed a clear inhibition when cerebellar stimulation preceded M1 stimulation of $8 \mathrm{~ms}$. However, conditioned responses with an ISI longer than $12 \mathrm{~ms}$ were also identified, showing mixed inhibitory and facilitatory effects. Responses showed anatomical selectivity for the intermediate hemispheric lobule IV-V-VI in the anterior cerebellum and for the midlateral portion of lobule VIIB in the posterior cerebellum. In

\section{MEP facilitation}

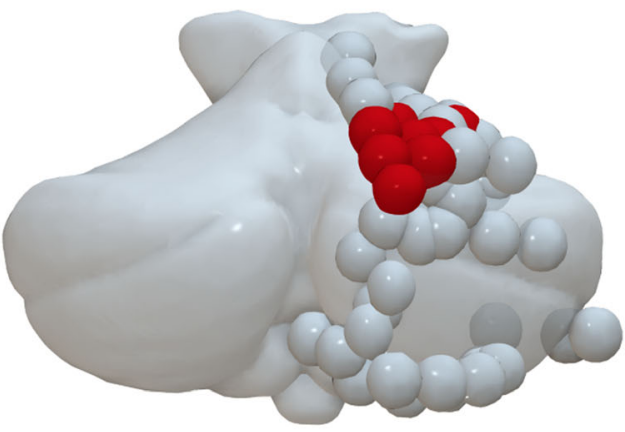

VIIB in the posterior cerebellum. The right panel indicates the locations where conditioning caused MEP facilitation, covering the intermediate lobule IV-V-VI in the anterior cerebellum. Blue-filled spheres indicate spots with significant inhibitory conditioning effects. Red-filled symbols indicate spots with significant excitatory conditioning effects. Grey-filled symbols indicate spots with no significant effect 
MEP conditioning between 8 and $16 \mathrm{~ms}$

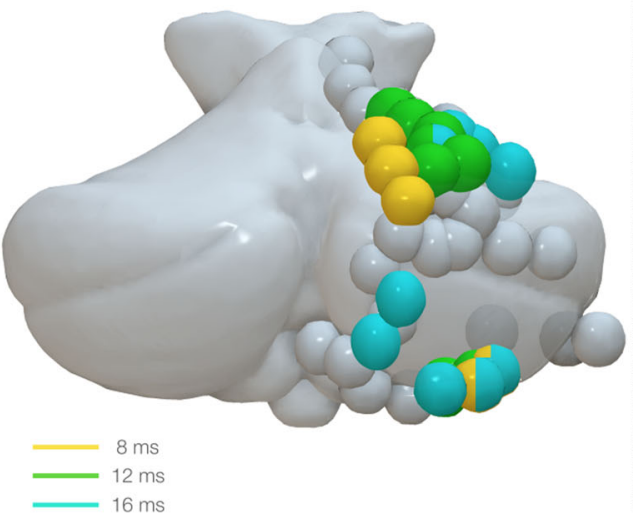

Fig. 6 Standardised cerebellar anatomy showing the location of each participant's conditioning electrodes in the cerebellar cortex. All stimulation sites have been displayed on the right hemisphere as spheres. The left panel shows with cerebellar location of dipoles with conditioning effect over M1 at ISI between 8 and $16 \mathrm{~ms}$. A mediolateral distribution was displayed in the anterior cerebellum, with

summary, we show that cerebello-cortical stimulation is feasible intraoperatively under general anaesthesia, with inhibitory responses at $8 \mathrm{~ms}$ likely representing the CDCT pathway. We speculate that using this cerebello-cortical paradigm during surgery could be used to predict, and potentially prevent, CDCT disconnection in children operated in the posterior fossa. This may help reduce postoperative cerebellar mutism and improve children's quality of life.

\section{Anatomical specificity for cerebello-cortical stimulation}

Dual coil TMS is a well-established technique for proving cerebellar function, which is characterised by MEP inhibition when cerebellar stimulation proceeds M1 stimulation about 5$8 \mathrm{~ms}$ [29]. To reproduce non-invasive stimulation results, we combined direct cerebellar stimulation with transcranical electrical stimulation using a 10-20 EEG setting [30], modifying a paradigm developed for supratentorial brain surgery [31]. MEP conditioning showed a very clear location specificity: MEP modification occurred when conditioning stimulations were performed in the intermediate lobule IV-V-VI in the anterior cerebellum and in the intermediate lobule VIIB in the posterior cerebellum. Strikingly, the anatomical location of our cerebellar maps corresponded with cerebellar activation for hand movement using fMRI found by Grodd and colleagues [32] in both the anterior and posterior cerebellum. Cerebellar stimulation caused MEP inhibition, reproducing established phenomena of the extraoperative setting [23, 24, 29]. However, areas of MEP facilitation were also shown. This is consistent with data of non-invasive protocols in humans, showing excitatory cerebellar effects on M1 depending on stimulation parameters [23] and with animal studies
MEP conditioning between 20 and 24 ms

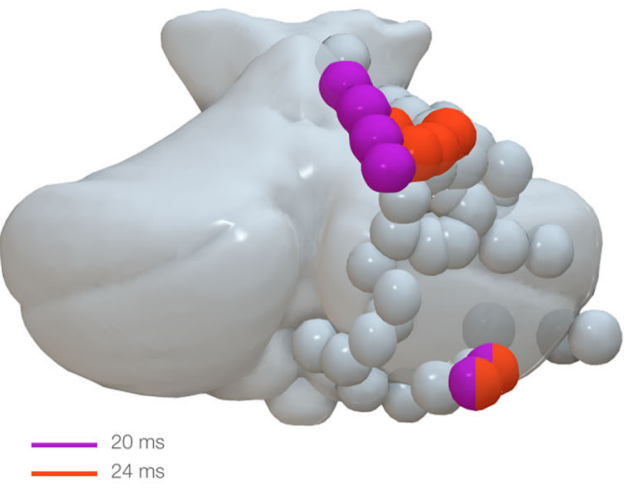

shorter ISI lying medially and longer ISI. Mixed distribution was shown in the inferior cerebellum. ISI at $8 \mathrm{~ms}$ : yellow dots; ISI at $12 \mathrm{~ms}$ : green dots; ISI at $16 \mathrm{~ms}$ : light blue dots. The right panel indicates as purple dots the locations where conditioning responses were evoked at $20 \mathrm{~ms}$ and orange dots at $24 \mathrm{~ms}$

showing that the cerebellum modulation would involve both cerebellar inhibition and excitation [33,34], since excitatory pathways exist that project to the cortex through the ventral thalamus [33]. Patterns of excitation and inhibition seemed to overlap in the anterior cerebellum, though inhibitory responses were more numerous and thus seemed more widespread. Facilitation was not evinced in the posterior cerebellum; however, it was less tested in our cohort compared with the anterior cerebellum. One issue that deserves discussion is the focality of cerebellar stimulation. We delivered bipolar stimuli through a dipole of $5 \mathrm{~mm}$ electrodes, $10 \mathrm{~mm}$ apart, at variable intensities, in the $10-25 \mathrm{~mA}$ range. We know from empirical and simulation data that inform us that stimuli in the cerebral cortex using these parameters produce electrical fields that are focal $[35,36]$. We can safely assume that the electrical field to bipolar stimulation is similar to that obtained by cerebral cortical stimulation; therefore, efficient neural stimulation did not occur in distant structures such as the cerebellar nuclei or the brainstem. However, it is uncertain how the electrical field translates into an electrical current and impacts the function of nervous tissue. Indeed, the cerebellar cortex shows striking anatomical differences from the cerebral cortex, including thinner cortical layers, deeper folding, dense stacking of adjacent folds and a much smaller radius of curvature of the surface. All these features add complexity to the problem of focality of stimulation. Given the complex folding pattern of the cerebellar cortex, it is likely that stimulation involves both cortical axons and efferent (Purkinje) axons in the white matter and that the cortex has been stimulated in an irregular and patchy pattern, with sparing of the deep part of the cerebellar sulci. A specific prediction on the local topography of stimulation can be made only by means of electricalfield modelling in anatomically realistic models of the 
cerebellar cortex, which are currently not available in the literature.

\section{Chronometry of cerebello-cortical stimulation}

Responses showed defined clusters which were ISI-related. In the anterior cerebellum, modulation showed a medio-lateral gradient, with $8 \mathrm{~ms}$ responses lying most medial and others lying progressively more lateral until $16 \mathrm{~ms}$. Moreover, responses at $24 \mathrm{~ms}$ lied lateral to those at $20 \mathrm{~ms}$. In the posterior cerebellum, conditioning responses had a more mixed distribution. Responses at $8 \mathrm{~ms}$ were always inhibitory in our cohort. This is consistent with the TMS literature, where cerebellar stimulation classically causes inhibition [29]. However, for all other intervals, inhibition nor facilitation was ISI-specific. This differs from Iwata and colleagues [37], where facilitation was associated with $2 \mathrm{~ms}$ conditioning only. As modulation from the cerebellum to the cerebrum should occur as fast as 5-8 $\mathrm{ms}$ [23], potentials occurring at $8 \mathrm{~ms}$ should represent the CDTC pathway. Responses at longer intervals (12-24 ms) may be consistent with other mechanism, possibly comprising activation of cerebello-spinal pathways [23]; however, further research is needed to elucidate involved connectivity.

\section{Cerebello-cortical stimulation and surgical relevance}

There is currently no method to monitor the CDTC pathway, considering that awake surgery is not indicated in pediatric patients [38], with 50-Hz stimulation technique being scarcely effective on children [39]. For the first time to our knowledge, we described this technique with the specific aim of providing a way to monitor the CDTC pathway and possible lower the incidence of CM. The present results are preliminary and exploratory. To capitalise on our results for the purpose of refining an intraoperative monitoring technique, it is probably better to focus on conditioning (cerebellar) stimuli applied to the intermediate lobule IV-V-VI in the anterior cerebellum or intermediate lobule VIIB, with ISIs around $8 \mathrm{~ms}$ or less (we did not manage to investigate ISIs $<8 \mathrm{~ms}$ in a systematic way), expecting purely inhibitory effects on the corticospinal motor pathway. Further studies using this method as a monitoring technique are needed to validate its reproducibility. In addition, the value of this potentially innovative neuromonitoring technique remains to be validated in the clinical setting, as this was not the goal of our feasibility study. We aim now to prospectively correlate neuromonitoring data (presence or absence of M1 output inhibition) with the clinical outcome in pediatric posterior fossa tumour surgery.

Finally, there is evidence that posterior fossa tumour resection can have different effects on children with pre-existing language impairment, which may represent a subclinical state of CMS in some children with posterior fossa tumour [40]. In this perspective, pre-operative TMS, following the protocol suggested by Ugawa and colleagues [23], may offer the opportunity to confirm the presence of a pre-surgical impairment of the CDTC pathway in these patients.

\section{Limitations}

There are several limitations to this study. First, the study collects both pediatric and adult patients: however, while CM is classically shown in children, CDTC is represented in both patients. Second, we could not monitor spinal excitability (e.g. with the H-reflex or F-waves) to distinguish whether conditioning occurring with a longer ISI represented cerebello-cortical or cerebello-spinal efferents. This issue should be addressed in further studies.

\section{Conclusion}

We showed for the first time that cerebello-cortical stimulation is a feasible technique to evidence cerebellar connectivity. While our results are compatible with previous literature on response chronometry, they also show a pronounced anatomical specificity for cerebellar modulation occurring in the midportion of hemispheric lobule IV-V-VI in the anterior cerebellum or in the second third of lobule VIIB, with both inhibitory but also excitatory effects evoked. These results may offer a new intraoperative technique for children suffering from posterior fossa tumour, with the aim to further contribute to prevent neurological deficits and hence to preserve quality of life.

Acknowledgements We wish to acknowledge Prof. A. Feletti, Dr. Francesco Cozzi and Dr. Fabio Moscolo for the valuable help in data collection. We wish to thank Henrietta Howells for the constructing comments on the manuscript.

Funding Open access funding provided by Università degli Studi di Verona within the CRUI-CARE Agreement.

\section{Declarations}

Conflict of interest The authors have no competing interests to declare.

Open Access This article is licensed under a Creative Commons Attribution 4.0 International License, which permits use, sharing, adaptation, distribution and reproduction in any medium or format, as long as you give appropriate credit to the original author(s) and the source, provide a link to the Creative Commons licence, and indicate if changes were made. The images or other third party material in this article are included in the article's Creative Commons licence, unless indicated otherwise in a credit line to the material. If material is not included in the article's Creative Commons licence and your intended use is not permitted by statutory regulation or exceeds the permitted use, you will need to obtain permission directly from the copyright holder. To view a copy of this licence, visit http://creativecommons.org/licenses/by/4.0/. 


\section{References}

1. Jones C, Baker SJ (2014) Unique genetic and epigenetic mechanisms driving paediatric diffuse high-grade glioma. Nat Rev Cancer 14:651-661

2. Baker SJ, Ellison DW, Gutmann DH (2016) Pediatric gliomas as neurodevelopmental disorders. Glia 64:879-895

3. Daly DD, Love JG (1958) Akinetic mutism. Neurology. 8:238-238

4. Pollack IF, Polinko P, Albright LA et al (1995) Mutism and pseudobulbar symptoms after resection of posterior fossa tumors in children: incidence and pathophysiology. Neurosurgery. 37: 885-892

5. Wells EM, Khademian ZP, Walsh KS et al (2010) Postoperative cerebellar mutism syndrome following treatment of medulloblastoma: neuroradiographic features and origin. J Neurosurg Pediatr 5: 329-334

6. Gudrunardottir T, Morgan AT, Lux AL et al (2016) Consensus paper on post-operative pediatric cerebellar mutism syndrome: the Iceland Delphi results. Childs Nerv Syst 32:1195-1203

7. De Smet HJ, Baillieux H, Catsman-Berrevoets C et al (2007) Postoperative motor speech production in children with the syndrome of "cerebellar" mutism and subsequent dysarthria: a critical review of the literature. Eur J Paediatr Neurol 11:193-207

8. De Smet HJ, Baillieux H, Wackenier P et al (2009) Long-term cognitive deficits following posterior fossa tumor resection: a neuropsychological and functional neuroimaging follow-up study. Neuropsychology 23:694-704

9. De Smet HJ, Paquier P, Verhoeven J, Mariën P (2013) The cerebellum: its role in language and related cognitive and affective functions. Brain Lang 127:334-342

10. Aarsen FK, Paquier PF, Reddingius RE et al (2006) Functional outcome after low-grade astrocytoma treatment in childhood. Cancer 106:396-402

11. Schmahmann JD (1996) From movement to thought: anatomic substrates of the cerebellar contribution to cognitive processing. Hum Brain Mapp 4:174-198

12. Stoodley CJ, Schmahmann JD (2009) Functional topography in the human cerebellum: a meta-analysis of neuroimaging studies. Neuroimage 44:489-501

13. Strick PL, Dum RP, Fiez JA (2009) Cerebellum and nonmotor function. Annu Rev Neurosci 32:413-434

14. Stoodley CJ, MacMore JP, Makris N et al (2016) Location of lesion determines motor vs. cognitive consequences in patients with cerebellar stroke. NeuroImage Clin 12:765-775

15. Cattaneo L, Fasanelli M, Andreatta O et al (2012) Your actions in my cerebellum: subclinical deficits in action observation in patients with unilateral chronic cerebellar stroke. Cerebellum 11:264-271

16. Van Baarsen KM, Grotenhuis JA (2014) The anatomical substrate of cerebellar mutism. Med Hypotheses 82:774-780

17. Catsman-Berrevoets CE, Van Dongen HR, Mulder PGH et al (1999) Tumour type and size are high risk factors for the syndrome of "cerebellar" mutism and subsequent dysarthria. J Neurol Neurosurg Psychiatry 67:755-757

18. Turgut M (1998) Transient "cerebellar" mutism. Childs Nerv Syst 14:161-166

19. Toescu SM, Hettige S, Phipps K et al (2018) Post-operative paediatric cerebellar mutism syndrome: time to move beyond structural MRI. Childs Nerv Syst 34:2249-2257

20. Nimsky C, Ganslandt O, Cerny S et al (2000) Quantification of, visualization of, and compensation for brain shift using intraoperative magnetic resonance imaging. Neurosurgery 47:1070-1080

21. D'Amico A, Sala F (2020) Intraoperative neurophysiology of the cerebellum: a tabula rasa. Childs Nerv Syst 36:1181-1186
22. Sloan T (2010) Anesthesia and intraoperative neurophysiological monitoring in children. Childs Nerv Syst 26:227-235

23. Ugawa Y, Day BL, Rothwell JC, Thompson PD, Merton PA, Marsden CD (1991) Modulation of motor cortical excitability. J Physiol 441:57-72

24. Hallett M, Di Iorio R, Rossini PM et al (2017) Contribution of transcranial magnetic stimulation to assessment of brain connectivity and networks. Clin Neurophysiol 128:2125-2139

25. Iwata NK, Ugawa Y (2005) The effects of cerebellar stimulation on the motor cortical excitability in neurological disorders: a review. Cerebellum 4:218-223

26. Shattuck DW, Leahy RM (2000) Brainsuite: an automated cortical surface identification tool. Lect Notes Comput Sci 1935:50-61

27. MacDonald DB (2006) Intraoperative motor evoked potential monitoring: overview and update. J Clin Monit Comput 20:347-377

28. Diedrichsen J (2006) A spatially unbiased atlas template of the human cerebellum. Neuroimage 33:127-138

29. Daskalakis ZJ, Paradiso GO, Christensen BK et al (2004) Exploring the connectivity between the cerebellum and motor cortex in humans. J Physiol 557:689-700

30. MacDonald DB, Skinner S, Shils J, Yingling C (2013) Intraoperative motor evoked potential monitoring - a position statement by the American Society of Neurophysiological Monitoring. Clin Neurophysiol 124:2291-2316

31. Cattaneo L, Giampiccolo D, Meneghelli P et al (2020) Corticocortical connectivity between the superior and inferior parietal lobules and the motor cortex assessed by intraoperative dual cortical stimulation. Brain Stimul 13:819-831

32. Grodd W, Hülsmann E, Lotze M et al (2001) Sensorimotor mapping of the human cerebellum: fMRI evidence of somatotopic organization. Hum Brain Mapp 13:55-73. https://doi.org/10.1002/ hbm. 1025

33. Allen GI, Tsukahara N (1974) Cerebrocerebellar communication systems. Physiol Rev 54:957-1006

34. Sniders RS, Magoun HW (1949) Facilitation produced by cerebellar stimulation. J Neurophysiol 12:335-345

35. Seo H, Kim D, Jun SC (2016) Effect of anatomically realistic fullhead model on activation of cortical neurons in subdural cortical stimulation-a computational study. Sci Rep 6:1-12. https://doi.org/ $10.1038 /$ srep27353

36. Yamamoto T, Katayama Y, Nagaoka T, Kobayashi K, Fukaya C (2004) Intraoperative monitoring of the corticospinal motor evoked potential (D-wave): clinical index for postoperative motor function and functional recovery. Neurol Med Chir 44(4):170-182

37. Iwata NK, Hanajima R, Furubayashi T et al (2004) Facilitatory effect on the motor cortex by electrical stimulation over the cerebellum in humans. Exp Brain Res 159:418-424. https://doi.org/10. 1007/s00221-004-1979-x

38. Gallentine WB, Mikati MA (2009) Intraoperative electrocorticography and cortical stimulation in children. J Clin Neurophysiol 26: 95-108. https://doi.org/10.1097/WNP.0b013e3181a0339d

39. Lohkamp LN, Mottolese C, Szathmari A et al (2019) Awake brain surgery in children - review of the literature and state-of-the-art. Childs Nerv Syst 35:2071-2077. https://doi.org/10.1007/s00381019-04279-w

40. Di Rocco C, Chieffo D, Frassanito P et al (2011) Heralding cerebellar mutism: evidence for pre-surgical language impairment as primary risk factor in posterior fossa surgery. Cerebellum 10:551562. https://doi.org/10.1007/s12311-011-0273-2

Publisher's note Springer Nature remains neutral with regard to jurisdictional claims in published maps and institutional affiliations. 